\title{
The World Space Observatory Ultraviolet (WSO-UV), as a bridge to future UV astronomy
}

\author{
B. Shustov ${ }^{1}$ - A.I. Gómez de Castro ${ }^{2}$ • \\ M. Sachkov ${ }^{1}$ • J.C. Vallejo ${ }^{2}$ • P. Marcos-Arenal ${ }^{2}$ • \\ E. Kanev ${ }^{1}$ - I. Savanov ${ }^{1}$ - A. Shugarov ${ }^{1}$ • \\ S. Sichevskii ${ }^{1}$
}

\begin{abstract}
The ultraviolet (UV) astronomy is a very demanded branch of space astronomy. Many dozens of short-term UV-experiments in space, as well as longterm observatories, have brought a very important knowledge on the physics and chemistry of the Universe during the last decades. Unfortunately, no large UV-observatories are planned to be launched by most of space agencies in the coming $10-15$ years. Conversely, the large UVOIR observatories of the future will appear not earlier than in 2030s. This paper briefly describes the projects that have been proposed by various groups. We conclude that the World Space Observatory - Ultraviolet (WSO-UV) will be the only $2-\mathrm{m}$ class UV telescope with capabilities similar to those of the HST for the next decade. The WSO-UV has been
\end{abstract}

\section{B. Shustov}

Institute of Astronomy of the RAS, Russia

A.I. Gómez de Castro

AEGORA Research Group, Universidad Complutense, Spain

M. Sachkov

Institute of Astronomy of the Russia

J.C. Vallejo

AEGORA Research Group, Universidad Complutense, Spain

P. Marcos-Arenal

AEGORA Research Group, Universidad Complutense, Spain

E. Kanev

Institute of Astronomy of the Russia

I. Savanov

Institute of Astronomy of the Russia

A. Shugarov

Institute of Astronomy of the Russia

S. Sichevskii

Institute of Astronomy of the Russia

${ }^{1}$ Institute of Astronomy, Russian Academy of Sciences,

Pyatnitskaya 48, 119017 Moscow, Russia

${ }^{2}$ AEGORA Research Group, Fac. CC. Matematicas, Universidad Complutense, Plaza de Ciencias 3, 28040 Madrid, Spain described in detail in previous publications, and this paper updates the main characteristics of its instruments and the current state of the whole project. It also addresses the major science topics that have been included in the core program of the WSO-UV, making this core program very relevant to the current state of the UV-astronomy. Finally, we also present here the ground segment architecture that will implement this program.

Keywords space vehicles, space vehicles: instruments, instrumentation: spectrographs, ultraviolet: general, ultraviolet: stars, ultraviolet: planetary systems, ultraviolet: ISM, ultraviolet: galaxies

\section{Introduction}

During almost half a century the astronomers have enjoyed a continuous access to the ultraviolet (UV) domain, $91.2-320 \mathrm{~nm}$, where the resonance transitions of the most abundant atoms and ions at temperatures between 3000 and $300000 \mathrm{~K}$ reside. Because this UVrange is not accessible from ground-based facilities, many short-term UV-experiments in space were carried out and a number of long-term space UV-observatories were put into orbit. They have brought a very important knowledge on the physics and chemistry of the Universe during the last decades.

Major achievements of UV-astronomy in this half a century are the following:

- Direct detection of $\mathrm{H}_{2}$ molecules in space with "Aerobee-150" (Carruthers (1970)).

- Discovery of the hot phase of the interstellar medium with "Copernicus" (Jenkins and Melov (1974)).

- Measurements of the D/H ratio with "Copernicus" (Rogerson and York (1973)). 
- Massive accurate determination of the chemical composition of stars and detailed studies of stellar mass loss phenomena across the HR diagram with the IUE mission $(1978$ - 1996).

- Identification of Warm-Hot Intergalactic Medium as the reservoir of missing baryons with HST and FUSE.

- Giant progress in understanding physics and chemistry of comets, planetary atmospheres and exospheres of exoplanets with HST.

All UV observations carried out from space, ranging from short-lived rocket and balloon-borned experiments, to small-aperture cameras and spectrographs on astrophysical and planetary missions, up to longlifetimes UV-observatories with powerful instruments on-board, have shown that UV-instruments on-board satellites bring great scientific benefits to the astronomical community. This makes natural a keen demand on new UV observatories.

The structure of this paper is as follows. In Section 2 we briefly review the current state of the art in UV astronomy, and discuss the ideas and concepts for future UV-observatories and instruments. In Section 3 we present current status of the World Space Observatory - Ultraviolet (WSO-UV) mission, which seems to be the only 2-m class UV telescope to fly in space in the next decade. Section 4 addresses the key science issues of this mission. The following section, Section 5, describes how the science program will be managed, and describes the current status of the WSO-UV ground segment. The last section, Section 6 , is devoted to the concluding remarks.

\section{On UV-instruments for astrophysics for today and tomorrow}

The telescopes for ultraviolet, optical and near-infrared ranges are commonly combined into the so-called UVOIR telescopes. This is because their optical design, detectors and general operational conditions (e.g. cryogenic temeratures are not required) are fundamentally similar. In this section discuss the current and future UVOIR facilities that are fully or partially intended for astrophysical observations in the near $(170-320 \mathrm{~nm})$ and far (91 - $180 \mathrm{~nm})$ UV wavelength range. Regarding the extreme UV, the effectiveness of instruments for astrophysical observations in the range $\lambda<91 \mathrm{~nm}$ is strongly limited by the high opacity of the interstellar medium, and after the EUVE (Extreme Ultraviolet Explorer) mission (Bowyer (1991)) there is no future significant project for observations beyond the Solar System in the extreme UV.
There is only one large UVOIR observatory currently flying in space, the Hubble Space Telescope (HST). In addition, there are three UV instruments on-board astrophysical observatories. The first instrument to consider is the Ultraviolet and Optical Telescope (UVOT), on-board the SWIFT satellite. UVOT has a $30 \mathrm{~cm}$ aperture that provides a $17^{\prime} \times 17^{\prime}$ field of view with a spatial resolution of $0.5 \%$ pixel in the optical/UV band (Roming et al. (2005)). The UVOT is based on the XMM-Newton mission's Optical Monitor instrument (Mason et al. (2001)), with improved optics and upgraded onboard processing computers. The third instrument is the Ultra Violet Imaging Telescope (UVIT) on-board ASTROSAT satellite. UVIT consists of twin 38-cm telescopes - one for the FUV region and the other for the NUV and visible (VIS) regions. UVIT is primarily an imaging instrument, simultaneously generating images in the FUV, NUV and VIS channels over a field of diameter 28 arcmin. Detailed description and review of the first results are presented in Subramaniam et al. (2016).

It is also of interest to report that the first China's lunar rover, launched in December 2013, is equipped with a $150 \mathrm{~mm}$ Ritchey-Chrétien telescope, called LUT (Lunar Ultraviolet Telescope). This telescope is being used to observe galaxies, active galactic nuclei, variable stars, binaries, novae, quasars and blazars in the near UV band (Wang et al. (2015)). It is capable of detecting objects at a brightness as low as magnitude 13, and the thin exosphere and slow rotation of the Moon allow extremely long, uninterrupted observations of the observed targets. Hence, the LUT can be considered the first long term lunar-based astronomical observatory.

Some other planetary missions are equipped with UV-instruments, which are in use in Target of Opportunity ( $\mathrm{ToO}$ ) mode. But we do not discuss here these missions, neither we will discuss the existing solar physics missions, confining ourselves to astrophysical projects.

As to the future a lot of ideas and projects are under discussion. Recently, Scowen et al. (2017) presented the science cases and technological discussions that came from the workshop titled "Finding the Ultraviolet-Visible Path Forward", held at NASA GSFC on June $25-26,2015$. The material presented there outlined the compelling science that can be enabled by a next generation of space-based observatories dedicated to UV-visible science, the technologies that are available for the design of those observatories, and the possible launch approaches to enhance the returned UV-science. A number of very large (apertures $>4 \mathrm{~m}$ ) UVOIR space telescopes have been proposed and intensively discussed during the last two decades. Any future UV/Optical telescope will require these large apertures to complement the very large IR and X-ray space 
telescopes and the $30 \mathrm{~m}$-class ground-based telescopes that will arrive in near future. Such large projects were considered at the Kavli IAU Workshop on "Global Coordination: Future Space-Based Ultraviolet-OpticalInfrared Telescopes", held in Leiden, the Netherlands, on July 17 - 19, 2017. The workshop sessions centered on 4 themes: (1) Setting the stage - astronomy in the 2030s, (2) Science drivers - why do we need a large UVOIR mission?, (3) Visions for large missions - what capabilities do we need?, and (4) How large missions fit into long-term space mission plan: 1 .

New technologies required for future large UV telescopes are not still all mature. The problem of very high cost remains unsolved either. It seems that the epoch for operational huge space UV-telescopes will come not earlier that in 2030s.

Despite of importance of UV observatories for the science progress, there are no consolidated plans in the programs of the major space agencies concerning even for mid-class UV-instruments for the next decade, but the the "World Space Observatory - Ultraviolet" (WSO-UV) project. WSO-UV seems to be the only $2 \mathrm{~m}$-class UV-telescope with capabilities similar to those of the HST that will guarantee access to the UV wavelength domain during the next decade. The WSO-UV is well developed. Therefore, it can be considered as a bridge from present facilities to future UV-astronomy obsevatories.

\section{The World Space Observatory - Ultraviolet mission}

The WSO-UV is a multi-purpose international space mission born as a response to the growing up demand for UV-facilities by the astronomical community. The nominal lifetime of this mission is 5 years, with a planned extension to cover 10 years of operations. The name "World Space Observatory - Ultraviolet" was introduced by Wamsteker (1999), and it reflects the international nature of the observatory. The project was initially considered by ESA and Roscosmos as a possible development of two successful UV projects, the IUE mission (Macchetto (1976); Nichols and Linskv (1996)) and the "Astron" mission Boyarchuk (1986, 1994). Nowadays, the WSO-UV implementation is led by the Federal Space Agency (Roscosmos, Russia), with Spain as a project partner. In the Federal Space Program, which is the major planning document for space activity in Russia, the project is also known as "SpektrUF", because the WSO-UV forms part of the "Spektr"

${ }^{1}$ The materials and results of the workshop are available at https://www.strw.leidenuniv.nl/KavliIAU2017 series of space missions. Besides the "Spektr-UF" the series includes the "Spektr-R", or "Radioastron" mission, launched in 2011, and the "Spektr-RG" mission, planned for launch in 2019. According to the Federal Space Program the launch of the WSO-UV is scheduled for 2023. We are to reserve up to six months for tests of the S/C systems and in-flight calibrations of scientific instruments.

The observatory will be put in a circular geosynchronous orbit with 51.6 degrees inclination; Earth occultation will be small and the orbital period will allow to time-track targets and to have rapid access to targets of opportunity. "Rapid" means that we can change program to the Director Discretionary Time Program (see below in Sect.5) in two hours.

The WSO-UV was described in detail in previous publications. See, for instance, Shustov et al. (2011, 2014); Sachkov et al. (2014, 2016). Therefore, we will just present here the most relevant new information and the latest updates to the project status. We will also present a brief overview of the major science topics that have been included in the Core Program of the WSO$\mathrm{UV}$, focusing on the recent suggestions for this program.

The funding in Russia for the WSO-UV project is guaranteed, and there are no critical technical problems in the implementation the project. The telescope is being planned for a launch from Baikonur (Kazakhstan) using a "Proton" rocket. The WSO-UV will use the Russian NAVIGATOR bus (platform), designed by Lavochkin Association. This platform has been already tested successfully in-flight by the "Radioastron" mission, and it has also been used by some commercial satellites.

The WSO-UV consists of a $1.7 \mathrm{~m}$ aperture RitcheyChrétien T-170M telescope (focal length $17 \mathrm{~m}$, field of view $30 \mathrm{arcmin}$ ). The mechanical part of this telescope has been manufactured by Lavockin Association, and it has successfully passed the required dynamic and thermal tests. The optics of the telescope has been manufactured by the Lytkarino optical plant, and both optics and coatings $\left(\mathrm{Al}+\mathrm{MgF}_{2}\right)$ meet the technical requirements. The AIV (Assembly Integration and Verification) facilities are almost ready to use, and the prefinal state of one of these AIV elements, an assembly stand for the T-170M telescope, is shown in Fig. 1.

The telescope is equipped with instrumentation designed to carry out high resolution spectroscopy, longslit low resolution spectroscopy and direct sky imaging. The main science instruments are the WSO-UV spectrographs (WUVS) and the Field Camera Unit (FCU).

The optical design of WUVS is presented in Panchuk et al. (2014). The WUVS consists of the following three channels (spectrographs): 
4

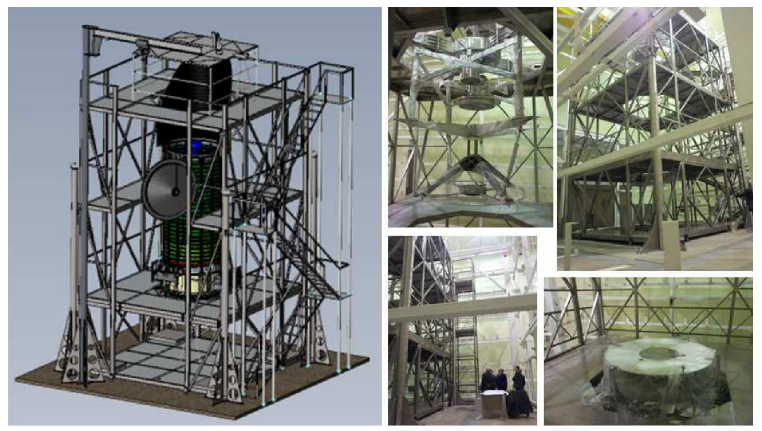

Fig. 1 The assembly stand of the T-170M telescope.

- The FUV high resolution spectrograph (VUVES), to carry out echellé spectroscopy with high resolution $(R \sim 50000)$ in the $115-170 \mathrm{~nm}$ range.

- The NUV high resolution spectrograph (UVES) to carry out echellé spectroscopy with $R \sim 50000$ in the $174-310 \mathrm{~nm}$ range.

- The Long Slit Spectrograph (LSS) that will provide low resolution $(R \sim 1000)$, long slit spectroscopy in the $115-305 \mathrm{~nm}$ range. The spatial resolution will be better than $0.5 \operatorname{arcsec}(0.1 \operatorname{arcsec}$ as the best value).

All spectrographs will be equipped with CCD detectors Shugarov et al. (2014). The Fig. 2 shows the Qualification Engineering Model of these CCD detectors.

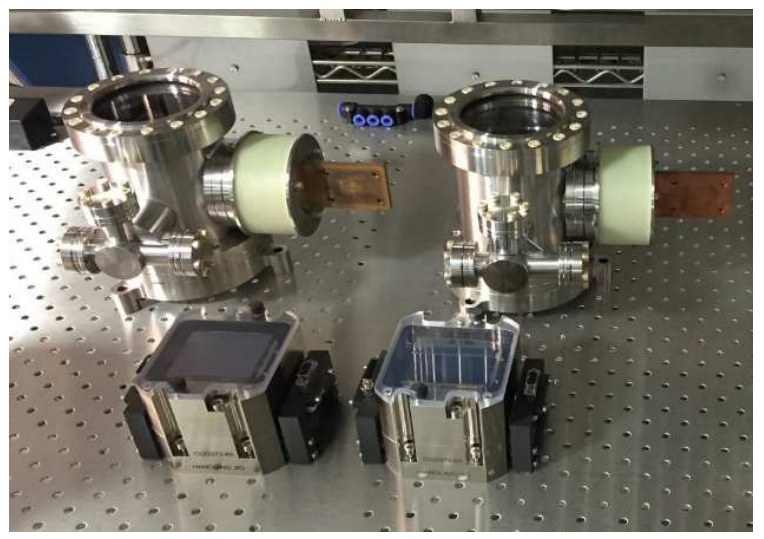

Fig. 2 Qualification Engineering Models of CCD detectors.

The spectral resolution provided by VUVES and UVES channels is similar to that offered by STIS/HST, but higher than the maximum resolution provided by COS/HST $(R \sim 20000)$.

The estimated effective area of UVES and VUVES channels of the WUVS is presented in Fig. 3. along the same parameters for the COS/HST and STIS/HST channels, for comparison purposes. Similarly, the estimated effective areas of the LSS channel of the WUVS, and the corresponding channels of the COS/HST are presented in Fig. 4

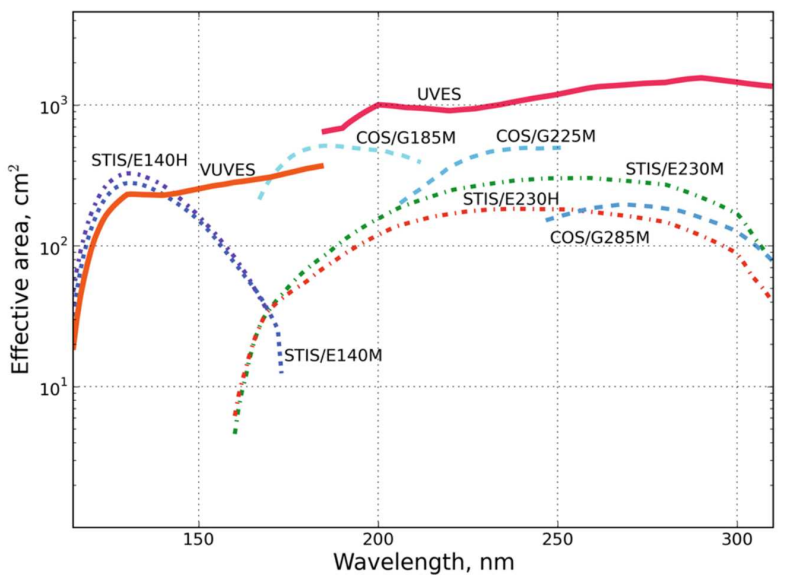

Fig. 3 The estimated effective area of UVES and VUVES channels of the WUVS, compared with the COS/HST and STIS/HST channels.

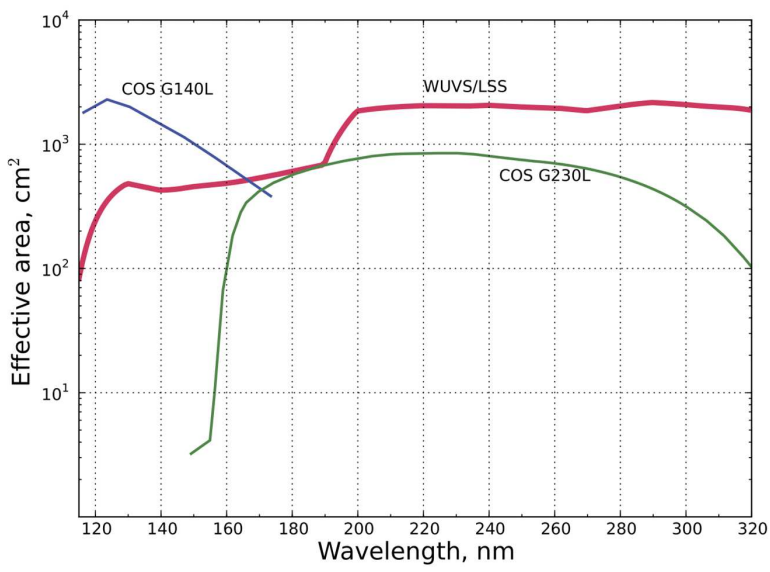

Fig. 4 The estimated effective area of the LSS channel of the WUVS, compared with the COS/HST channels.

The imaging functions of the WSO-UV were initially assigned to the Imaging and Slitless Spectroscopy Instrument (ISSIS), under responsibility of Spain Gómez de Castro et al. (2014), designed to carry out imaging and slitless spectroscopy in the 115 $320 \mathrm{~nm}$ spectral range. The ISISS was to be equipped with two MCP detectors, with CsI and CsTe photocathods for FUV and NUV observations, respectively. The capabilities of the ISSIS was expected to be similar to those of the Advanced Camera for Surveys of the HST (ACS/HST).

This scenario has changed, and now the imaging capabilities for the WSO-UV are fulfilled by the Field Camera Unit (FCU), the successor to ISSIS. The FCU will be the first UV-imager to be flown to a geosynchronous orbit above the Earth geocorona. The main task of the FCU is to obtain high resolution images and low resolution spectra in FUV and NUV bands 
Sachkov et al. (2017). The Fig. 5 shows the preliminary design of FCU.

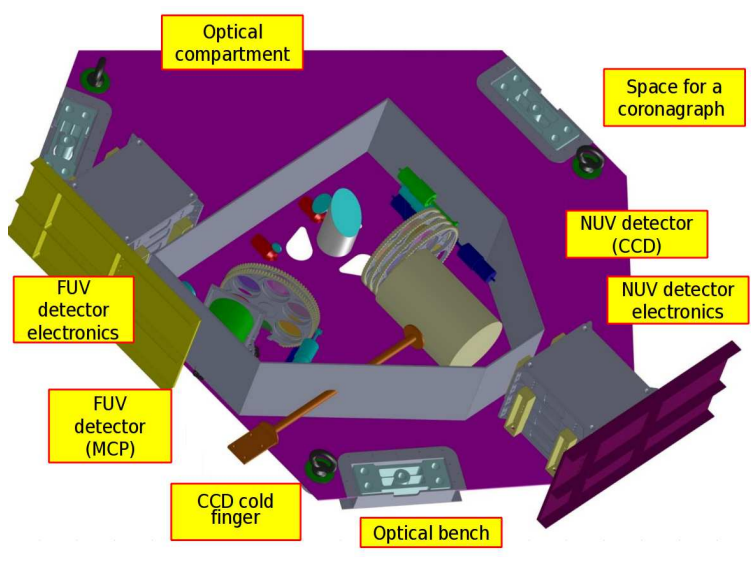

Fig. 5 Preliminary design of the FCU.

The FUV channel is equipped with a MCP detector sensitive in $115-176 \mathrm{~nm}$ range. The main characteristics of this channel are:

- Diffraction-limited image quality in FUV.

- High sensitivity photon-counting mode.

- High time resolution.

The NUV channel is equipped with a CCD detector sensitive in $174-310$ (extended to 1000) nm range. The main characteristics of this channel are:

- Wide field of view.

- High dynamic range.

- Possibility of low resolution field spectroscopy (provided by grism).

The Table 1 shows the main features of the FCU channels. This table also presents the corresponding features of HST/ACS/SBC and HST/WFC3/UVIS, taken from the HST Instruments Handbook, for comparison purposes.

\section{WSO-UV Key Science Issues}

The present day hot science topics should constitute the core program of any space project, and WSO-UV is not an exception. It is important to focus the science program of any mission on the most challenging science issues that can be most efficiently studied with the observatory instruments. As these science issues evolve with time, any project needs to continuously adapt the mission priorities to the most actual topics. The science case of the UV astronomy, and that of WSO-UV in particular, has been discussed recently in Boyarchuk et al. (2016).
Currently, the Core Program of the WSO-UV includes:

- The determination of the diffuse baryonic content in the Universe and its chemical evolution. The main topics will be the investigation of baryonic content in Warm-Hot Inter Galactic Matter (WHIM) and the Ly- $\alpha$ systems.

- The study of the formation and evolution of galaxies.

- The physics of accretion and outflows: stars, black holes, and all those objects dominated by accretion mechanisms. The efficiency and time scales of the phenomena will be studied, together with the role of the radiation pressure and the disk instabilities.

- The investigation of the extrasolar planetary atmospheres and astrochemistry in presence of strong UV radiation fields.

\subsection{The baryonic content of the IGM}

As it was noted by Nicastro (2014), baryons are missing at all astronomical scales in Universe. Independent of the different models for the evolution of the Universe, the major baryonic component of the Universe must be associated with the intergalactic medium (IGM). Hydro-dynamical simulations for the formation of structures tend to re-concile the different "missingbaryon" problems and predict that most of the baryonic matter of the Universe is hiding in a hot and tenuous gaseous phase. Bregman et al. (2015) argues that about $30-50 \%$ of the baryons in the local Universe are likely in a hot phase and Warm-Hot Inter Galactic Matter (WHIM), $T=10^{5}-10^{8} \mathrm{~K}$, and the dominant ions have their ground state resonance transitions in the UV and X-ray domains.

Share of missing baryons in the local Universe $(z \leq$ 2 ) is almost two times larger than that in the more distant Universe (Nicastro (2014)). This is because the distant objects, which emit/absorb in UV, are observable in optical range due to cosmological shift.

Thus UV emiting/absorbing plasma (WHIM and Ly $\alpha$-absorbers) is a major reservoir of missing baryons. Observation of broad hydrogen Ly $\alpha$ absorption is one of the most promising techniques to map the distribution of the WHIM.

\subsection{The baryons in DM halo and formation of galaxies.}

The models of the formation of galaxies like our Milky Way have predicted that the central galaxy only contains a modest fraction of the available baryons (Klypin et al. (2011)). Galaxies are inefficient producers that have converted a small portion of their available gas into stars. It has become increasingly apparent 
Table 1 Main features of the WSO-UV FCU, compared with those from HST/ACS/SBC and HST/WFC3/UVIS

\begin{tabular}{lrrrr}
\hline Parameters & FUV Channel & NUV Channel & HST/ACS/SBC & HST/WFC3/UVIS \\
\hline Detector & MCP & CCD & MCP, MAMA & CCD \\
Spectral range, nm & $115-170$ & $174-310$ (ext. to 1000) & $115-170$ & $200-1000$ \\
Effective area, m & 0.068 & 0.27 & 0.18 & 0.45 \\
Field of view, arcsec & $121 \times 121$ & $597 \times 451$ & $35 \times 31$ & $162 \times 162$ \\
Scale, arcsec/pixel & $0.08^{[a]}$ & 0.146 & $0.033 \times 0.030$ & 0.0395 \\
Detector size, mm & 30 & $49 \times 37$ & 25 & $61 \times 61$ \\
Detector format & $2 \mathrm{k} \times 2 \mathrm{k}$ & $4 \mathrm{k} \times 3 \mathrm{k}$ & $1 \mathrm{k} \times 1 \mathrm{k}$ & $1 \mathrm{k} \times 1 \mathrm{k}$ \\
Number of filters & up to $10+2$ prisms & up to 15 & $6+2 \mathrm{prism}$ & 62 \\
\hline
\end{tabular}

[a] Scale is tentative.

over the past years that galaxies also exhibit a diffuse baryonic component within the dark matter halo that extends far from the inner regions to the virial radius and beyond. This gas is a target of COS-Halos Project (HST). Werk et al. (2014) analyzed the physical conditions of the cool, photoionized $\left(T \sim 10^{4} \mathrm{~K}\right)$ circumgalactic medium (CGM) using the COS-Halos suite of gas column density measurements for 44 gaseous halos within $160 \mathrm{kpc}$ galaxies at $z \sim 0.2$, and the WSO-UV is believed to be an efficient instrument to contribute to the inventory of baryons in the galactic vicinity.

\subsection{Astronomical engines}

The astronomical engines (stars, black holes, etc...) can accelerate large masses to velocities close to the speed of light or generate sudden ejections of mass as those observed in Supernova explosions. The WSO-UV will provide key inputs to answer the fundamental open questions concerning the physics of these objects. Some few examples are:

1. High resolution UV spectroscopy will allow to determine the structure of the accretion flow on magnetic cataclismic variables and on $\mathrm{T}$ Tauri stars, and to measure the physical conditions and clumpyness of the outflows. It will also allow to study the source of the energy that powers the extended dense $\left(\geq 10^{10} \mathrm{~cm}^{-3}\right)$ and hot $\left(\mathrm{T}_{e} \geq 60,000 \mathrm{~K}\right)$ envelopes with luminosities about $0.2 \mathrm{~L}_{\odot}$ that have been detected around T Tauri stars (Gomez de Castro et al. (2003)).

2. Low resolution spectroscopy will allow to measure the general physical conditions and metalicities of the Broad Lines Emission Region in Active Galactic Nuclei. Reverberation mapping will allow to study the kinematics and the mass of the central supermassive black holes. The atmospheres of the hot accretion disks in cataclysmic variable stars and the role of disk instabilities in triggering the outbursts will be also studied.
3. The high sensitivity of the FUV camera will allow to detect hot jets through their Ly- $\alpha$ and to resolve the thermal structure of the jets and the regions shocked by them. It will also allow to survey star forming regions to detect planetary-mass objects in regions like $\sigma$-Orionis and to study the magnetic activity and accretion processes in these free-floating planetarylike objects which are at the low mass end of the molecular clouds fragmentation scales.

\subsection{Extrasolar planetary atmospheres}

The instrumentation of the WSO-UV project is very important and helpful for exoplanet studies and for the characterization of the exoplanet-stellar environment.

There are still several difficulties that are at the origin of the major uncertainties on any estimation of exoplanetary atmosphere properties (Fossati et al. (2014)):

- The relative faintness of the UV stellar emissions.

- The variability of the sources.

- The signal contamination by both the sky background signal (at some spectral lines) and the instrument response.

While the first difficulty can be solved by focusing on a few close-by and UV-bright stars, the signal variability from both the source and the instrument is a real problem that should be addressed to build a reliable diagnostic to extract an accurate description of the upper atmosphere and of the interaction region between the exoplanet and the impinging wind from its host star.

In addition to standard exoplanet observations and characterizations, the WSO-UV observatory can be also used for observations of biomarkers. The biomarkers like ozone have very strong transitions in the ultraviolet. These are electronic molecular transitions, hence several orders of magnitude stronger than the vibrational or rotational transitions observed in the infrared or radio range. The spectral resolving power required to detect biomarkers in the atmosphere of exoplanets is not a crucial capability. A resolution of $R \sim 10000$ is 
adequate for these investigations, and even $R \leq 1000$ could be enough to detect the broad band signatures of many molecules. The presence of biomarkers and other constituents in the atmospheres can be searched by the WSO-UV high resolution spectrographs for hundreds of exoplanets orbiting $\mathrm{K}, \mathrm{G}$ and $\mathrm{F}$-type main sequence stars.

\subsection{Physics of stars as progenitors of some GW events}

Hamann et al. (2017) noted that the recent discovery of a gravitational wave from the merging of two black holes, of about 30 solar masses each one, challenges our incomplete understanding of massive stars and their evolution. The UV-observations are critical for studying the physics of these massive stars. Critical ingredients comprise mass-loss, rotation, magnetic fields, internal mixing, and mass transfer in close binary systems. The imperfect knowledge of these factors implies large uncertainties when modeling the stellar populations and their feedback. Wang and Huang (2017) describes the UV-behaviour of GW 170817 and concludes that the soft X-ray/UV emission could provide the earliest localization of the corresponding electromagnetic counterparts of the gravitational waves from double neutron star mergers.

have rapid access to targets of opportunity.

\section{Management of the science program and the architecture of the ground segment of the WSO-UV mission}

The aim of the WSO-UV project is the creation of an international observatory working in the UV range which is inaccesible for Earth-based instruments. The WSOUV as international observatory will make regular Announcements of Opportunity to the scientific community for observing time to be solicited by international teams. The WSO-UV is expected to carry over three main scientific research programs:

- The Core Program (CP) that includes the key scientific projects driving the development of the WSOUV mission.

- The National Programs (NP) that grant a fraction of the observing time to the Countries involved in the WSO-UV development.

- The Open Program (OP) to the world-wide scientific community.

Additional programs are:

- The Director Discretionary Time Program, that represents a small fraction of the observatory time and will be managed by the director of the WSO-UV observatory to allow a rapid response to unexpected important astronomical events or for other scientific purposes.

- The Calibration Program, that includes specific observations and measurements to calibrate and ensure the optimal performance of the WSO-UV.

- The Guaranteed Time Program for the Instrument Teams.

Only excellent proposals will be eligible for the "Core Program" (CP) but there are no limitations in the amount of time or instrumentation. The proposals to all the core program and remaining observing programs will be submitted to the Science Operations Center (SOC) for evaluation of their scientific excellence and technical feasibility. Indeed, it is foreseen that there will be two initial calls devoted to just implement the core program of the mission, well before the launch date. The first CP call will be devoted to those proposals requiring observing time with other facilities (preparatory observations), and the second CP call will be devoted to those proposals not requiring any preparatory observation, and to those that participated in the first call.

Therefore, the elements of the ground segment in charge of handling these early calls must be already developed and implemented in time, well ahead the launch date. Because of this staggered schedule, an incremental and iterative strategy for implementing the necessary parts of the ground segment has been set in place.

The WSO-UV ground segment is composed of all the infrastructure and facilities involved in the preparation and execution of the WSO-UV mission operations. These facilities include all equipment and services needed for the mission preparation and to perform the actual operations: Ground Stations, Mission Control Centre, Science Control Centre, Instrument Teams, etc.

Functionally, the ground segment comprises downlink and uplink systems. The downlink part encompass the systems in charge of the monitoring and control of the spacecraft, telescope and instrument, as well as reception, processing and storage of the scientific data. The uplink chain includes those systems in charge of the reception of proposals for observing from the users of the telescope, the systems in charge of planning the sequences of observations and the systems in charge of final conversion into telecomands to be uploaded to the spacecraft.

This high-level architecture has been refined as the project has evolved. Previous reports on its progress can be found in Malkov et al. (2011); Gómez de Castro et al. (2013). This architecture splits the ground segment in two "standard" elements, a Mission Operations Centre 
(MOC), and a Science Operations Center (SOC). However, some simplifications have been recently done to the existing baseline, aiming to reduce the costs and increase the scientific return of the mission.

The Mission Operations Centre (MOC) includes all technical systems, operational procedures and manpower related to the provision of the spacecraft status analyses, spacecraft functioning control, monitoring of the platform, overall payload and ground segment. This includes commanding capabilities for the platform, payload and ground segment, control procedure generation and execution, performance evaluation, low-level mission planning sequences generation, on-board software management and maintenance and operational database management systems.

The Science Operations Centre (SOC) includes all technical systems, operational procedures and manpower available required for supporting the planning, monitoring and control, and performance evaluation of the payload elements on board the space segment. The SOC activities cover the science operation planning activities, such as proposal reception and handling, and creation of a timeline of observations to be uplinked to the satellite (uplink related responsibilities), and it also covers the science data operations, such as science data processing and archiving (downlink related responsibilities).

The initial baseline for the ground segment was based on two complete Missions Operations Centres (MOC), and two complete Science Operations Centers (SOC), both sharing the operations on a regular basis. A prototype of this integrated dual ground segment was developed and tested during $2012-2013$. As a consequence of the test campaign, a re-engineering process took place, aiming to simplify the operations, maximize the available resources and reuse the existing multi-mission MOC facilities at Russian side.

The WSO-UV ground segment baseline now relies in one single MOC, located in Moscow (Lavochkin Science and Technology Association). This single entity will be the unique interface between the ground segment and the spacecraft in which concerns the low-level telecommanding capabilities, fully under Russian responsibility.

The concept of sharing operations remains for the science-side of the WSO-UV operations, under SOC responsibility. The sharing of science operations is kept by building a modular SOC, where some elements are unique, meanwhile others can be duplicated. This allows the reuse of the prototyped architecture and components, but also the removal of a heavy common MOCSOC infrastructure.

The SOC is responsible for the quality of the science mission products and for the scientific performance of

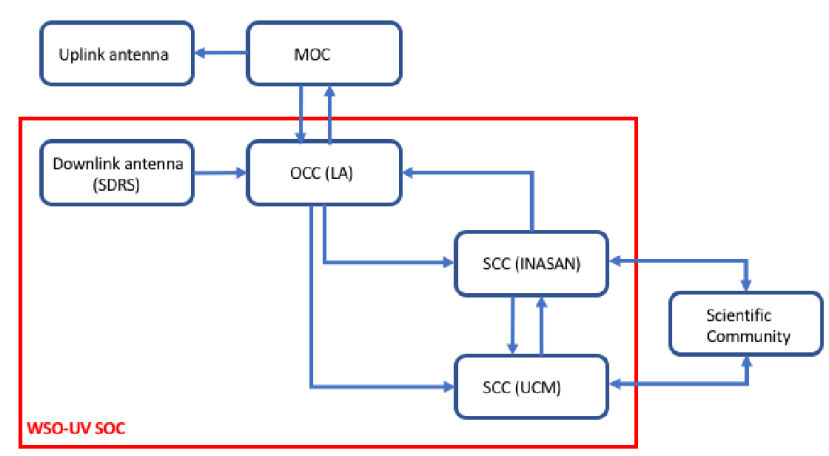

Fig. 6 WSO-UV SOC context within the mission ground segment. LA=Lavochkin. INASAN=Institute of Astronomy of the Russian Academy of Science. UCM=University Complutense of Madrid

the mission. Therefore, its functionalities include the interaction with the Mission Operations Centre (MOC), instrument calibration, long term planning (integrated science schedule), science data archiving, and interaction with the mission external users. The main SOC elements within the Ground Segment are seen in Figure 6. The SOC combines at the same time an uplink function and a downlink function. Therefore, it is split in a Scientific Data Reception System (SDRS), an Operational Control Centre (OCC), and two Science Control Centres (SCCs).

The SDRS, referred in Russian as "Apparatus for Reception of Scientific Data", is formed by the set of mission available antennas dedicated to reception of the signal from satellite and sending the downlink TM data (in L0 format) to the MOC and to the Operational SOC.

\section{ANA's EXPLANATION FOR L1, L1, L2}

The OCC, referred in Russian as "Center of Scientific Data Reduction", is the part of the SOC co-located with the MOC, and it is formed by the systems in charge of creating the low-level sets of sequences that will be converted into $\mathrm{TC}$ and uplinked to the S/C by the MOC, receiving the TM data from the SDRS and performing a preliminary data reduction (Quick Look Analysis) and sending the downlink TM and Aux data needed for scientific processing to the SCC.

The SCCs, referred in Russian as "Russian/Spanish Regional SOC", are formed by the set of SOC components in charge of the final archive of data, the L2 processing from L1 level, the provision of the scientific analysis tools to the community, the provision of proposal reception and handling systems.

There will be two SCCs, one at the Institute of Astronomy of the Russian Academy of Science (INASAN), in Moscow, Russia, and one at the Joint Center for 
Ultraviolet Astronomy (JCUVA), located in University Complutense of Madrid, Spain.

Every SCC has the following components:

- The Mission Data Archive System (MDA), main data repository and index to the WSO-UV mission.

- The Science Data Pipeline System (SDP), in charge of processing L1 data product as received from dedicated Downlink Antennas to obtain mission L2 products.

- The Science Control System (SCS), in charge of handling the communications and data interchange between the SCC INASAN and the SCC UCM., and the reception of the TM and Aux Data coming from the OCC.

- The Sequence Generator System (SGS, only at INASAN), that converts the lists of targets approved by the Time Allocation Committee of the project into low-level sequences of time-tagged set of observations that will be sent to the OCC for later creation of TCs to be uplinked.

- A set of Interfaces and Scientific Analysis Tools (the IAS), formed by SCC Simulators, Proposal Tools, MDA Access tools and general Community Support Tools.

The SOC conforms one of the major parts of the Spanish contributions to the mission. It is been built in a modular and incremental way in order to fullfil the requirements of the mission as they are needed. The SCC will be the first operational element of the Ground Segment to be implemented, because it will support the core program call to take place well before the launch date of the spacecraft.

The different components of the SCC are defined and developed by a international Science Team composed of the Spanish and Russian Science Support Teams based at the Universidad Complutense of Madrid (UCM) and the Institute of Astronomy of the Russian Academy of Science (INASAN). This Science Team provides all technical systems and manpower available required for supporting the operation and processing of the Scientific Instruments on-board the WSO-UV spacecraft. Therefore, it is responsible for laying the foundation of and supervising all the operations related to the mission primary users: the scientists. At mission level, the Science Team constitutes the core of the future WSO-UV observatory.

\section{Concluding remarks}

The UV-astronomy is a very demanded branch of space astronomy. Many dozens of short-term UVexperiments in space as well as long-term space observatories have brought very important knowledge on physics and chemistry of the Universe. A number of very large (aperture $>4 \mathrm{~m}$ ) UVOIR space telescopes have been intensively proposed and discussed during the last two decades. Unfortunately, none of these large UV-observatories is planned to be launched in the coming $10-15$ years by most of space agencies. Therefore, large UVOIR observatories of the future will not appear earlier than in 2030s. The World Space Observatory Ultraviolet (WSO-UV) will be the only $2 \mathrm{~m}$ class UVtelescope with capabilities similar to the HST ready to fly for the next decade. The WSO-UV will work as a space targeted observatory with a core program and an open program for scientific projects from the world-wide community and national (funding) bodies (Malkov et al. (2011)). The core program of the WSOUV remains very relevant to the current state of the UV-astronomy. The latest information on this project can be found at the official web site http://wso-uv.org.

Acknowledgements B. Shustov and E. Kanev thank the Russian Science Foundation for supporting this work by grant RSF 17-12-01441. Ana I. Gómez de Castro, Juan C. Vallejo and Pablo Marcos-Arenal thank the Spanish Ministry of Economy, Industry and Competitiveness for grants ESP2014-54243-R and ESP201568908-R. 


\section{References}

Bowyer, S.: In: Bulletin of the American Astronomical Society. Bull. Am. Astron. Soc., vol. 23, p. 972 (1991)

Boyarchuk, A.A.: Irish Astronomical Journal 17, 352 (1986)

Boyarchuk, A.A.: Astrophysical Research with the Space Station Astron (rus. Astrofizicheskie Issledovania na Kosmicheckoi Stantsii Astron), Nauka Press, Moscow, 1994 (isbn 5-02-014515-7), (1994)

Boyarchuk, A.A., Shustov, B.M., Savanov, I.S., Sachkov, M.E., Bisikalo, D.V., Mashonkina, L.I., Wiebe, D.Z., Shematovich, V.I., Shchekinov, Y.A., Ryabchikova, T.A., Chugai, N.N., Ivanov, P.B., Voshchinnikov, N.V., Gomez de Castro, A.I., Lamzin, S.A., Piskunov, N., Ayres, T., Strassmeier, K.G., Jeffrey, S., Zwintz, S.K., Shulyak, D., Gérard, J.-C., Hubert, B., Fossati, L., Lammer, H., Werner, K., Zhilkin, A.G., Kaigorodov, P.V., Sichevskii, S.G., Ustamuich, S., Kanev, E.N., Kil'pio, E.Y.: Astronomy Reports 60, 1 (2016)

Bregman, J.N., Alves, G.C., Miller, M.J., Hodges-Kluck, E.: Journal of Astronomical Telescopes, Instruments, and Systems 1(4), 045003 (2015). 1506.03469

Carruthers, G.R.: Astrophys. J. Lett. 161, 81 (1970). doi:10.1086/180575

Fossati, L., Bisikalo, D., Lammer, H., Shustov, B., Sachkov, M.: Astrophys. Space Sci. 354, 9 (2014)

Gomez de Castro, A.I., Verdugo, E., Ferro-Fontan, C.: In: Brown, A., Harper, G.M., Ayres, T.R. (eds.) The Future of Cool-Star Astrophysics: 12th Cambridge Workshop on Cool Stars, Stellar Systems, and the Sun. Cambridge Workshop on Cool Stars, Stellar Systems, and the Sun, vol. 12, p. 40 (2003)

Gómez de Castro, A.I., Sestito, P., Sanchez Doreste, N., López-Martínez, F., Seijas, J., Rodríguez, P., Gómez, M., Lozano, J.M., Shustov, B., Sachkov, M., Moisheev, A.: In: Guirado, J.C., Lara, L.M., Quilis, V., Gorgas, J. (eds.) Highlights of Spanish Astrophysics VII, p. 820 (2013)

Gómez de Castro, A.I., Sestito, P., Sánchez, N., LópezMartínez, F., Seijas, J., Gómez, M., Rodríguez, P., Quintana, J., Ubierna, M., Muñoz, J.: Advances in Space Research 53, 996 (2014). 1302.1004

Hamann, W.-R., Oskinova, L., Todt, H., Sander, A., Hainich, R., Shenar, T., Ramachandran, V.: In: Eldridge, J.J., Bray, J.C., McClelland, L.A.S., Xiao, L. (eds.) The Lives and Death-Throes of Massive Stars. IAU Symposium, vol. 329, p. 223 (2017). 1702.05629. doi:10.1017/S1743921317002563

Jenkins, E.B., Meloy, D.A.: Astrophys. J. Lett. 193, 121 (1974). doi:10.1086/181647

Klypin, A.A., Trujillo-Gomez, S., Primack, J.: Astrophys. J. 740, 102 (2011). 1002.3660. doi:10.1088/0004$637 \mathrm{X} / 740 / 2 / 102$

Macchetto, F.: Mem. Soc. Astron. Italiana 47, 431 (1976)

Malkov, O., Sachkov, M., Shustov, B., Kaigorodov, P., Yáñez, F.J., Gómez de Castro, A.I.: Astrophys. Space Sci. 335, 323 (2011). doi:10.1007/s10509-010-0589-2

Mason, K.O., Breeveld, A., Much, R., Carter, M., Cordova, F.A., Cropper, M.S., Fordham, J., Huckle, H. Ho, C., Kawakami, H., Kennea, J., Kennedy, T., Mittaz, J., Pandel, D., Priedhorsky, W.C., Sasseen, T.,
Shirey, R., Smith, P., Vreux, J.-M.: Astron. Astrophys. 365, 36 (2001). astro-ph/0011216. doi:10.1051/00046361:20000044

Nicastro, F.: In: The X-ray Universe 2014, p. 11 (2014)

Nichols, J.S., Linsky, J.L.: Astron. J. 111, 517 (1996). doi:10.1086/117803

Panchuk, V., Yushkin, M., Fatkhullin, T., Sachkov, M.: Astrophys. Space Sci. 354, 163 (2014). doi:10.1007/s10509014-2087-4

Rogerson, J.B., York, D.G.: Astrophys. J. Lett. 186, 95 (1973). doi:10.1086/181366

Roming, P.W.A., Kennedy, T.E., Mason, K.O., Nousek, J.A., Ahr, L., Bingham, R.E., Broos, P.S., Carter, M.J., Hancock, B.K., Huckle, H.E., Hunsberger, S.D., Kawakami, H., Killough, R., Koch, T.S., McLelland, M.K., Smith, K., Smith, P.J., Soto, J.C., Boyd, P.T., Breeveld, A.A., Holland, S.T., Ivanushkina, M., Pryzby, M.S., Still, M.D., Stock, J.: Space Sci. Rev. 120, 95 (2005). astro-ph/0507413. doi:10.1007/s11214-005-5095-4

Sachkov, M., Shustov, B., Gómez de Castro, A.I.: In: Space Telescopes and Instrumentation 2016: Ultraviolet to Gamma Ray. Proc. SPIE, vol. 9905, p. 990504 (2016). doi:10.1117/12.2233085

Sachkov, M., Shustov, B., Savanov, I., Gómez de Castro, A.I.: Astronomische Nachrichten 335, 46 (2014). doi:10.1002/asna.201312015

Sachkov, M., Shustov, B., Gómez de Castro, A.I., Shugarov, A., Savanov, I., Kanev, E., Sichevskij, S., Zvereva, M.: In: Balega, Y.Y., Kudryavtsev, D.O., Romanyuk, I.I., Yakunin, I.A. (eds.) Stars: From Collapse to Collapse. Astronomical Society of the Pacific Conference Series, vol. 510 , p. 573 (2017)

Scowen, P.A., Tripp, T., Beasley, M., Ardila, D., Andersson, B.-G., Maíz Apellániz, J., Barstow, M., Bianchi, L., Calzetti, D., Clampin, M., Evans, C.J., France, K., García García, M., Gomez de Castro, A., Harris, W., Hartigan, P., Howk, J.C., Hutchings, J., Larruquert, J., Lillie, C.F., Matthews, G., McCandliss, S., Polidan, R., Perez, M.R., Rafelski, M., Roederer, I.U., Sana, H., Sanders, W.T., Schiminovich, D., Thronson, H., Tumlinson, J., Vallerga, J., Wofford, A.: Publ. Astron. Soc. Pac. 129(7), 076001 (2017). 1611.09736. doi:10.1088/1538$3873 / 129 / 977 / 076001$

Shugarov, A., Savanov, I., Sachkov, M., Jerram, P., Moody, I., Pool, P., Turner, P., Pittock, R., Kuzin, S., Waltham, N.: Astrophys. Space Sci. 354, 169 (2014). doi:10.1007/s10509-014-1911-1

Shustov, B., Sachkov, M., Gómez de Castro, A.I., Werner, K., Kappelmann, N., Moisheev, A.: Astrophys. Space Sci. 335, 273 (2011). doi:10.1007/s10509-011-0737-3

Shustov, B., Gómez de Castro, A.I., Sachkov, M., Moisheev, A., Kanev, E., López-Santiago, J., Malkov, O., Nasonov, D., Belén Perea, G., Sánchez, N., Savanov, I., Shugarov, A., Sichevskiy, S., Vlasenko, O., Yañez, J.: Astrophys. Space Sci. 354, 155 (2014). doi:10.1007/s10509-014-21190

Subramaniam, A., Tandon, S.N., Hutchings, J., Ghosh, S.K., George, K., Girish, V., Kamath, P.U., Kathiravan, S., Kumar, A., Lancelot, J.P., Mahesh, P.K., Mohan, R., Murthy, J., Nagabhushana, S., Pati, A.K., 
Postma, J., Rao, N.K., Sankarasubramanian, K., Sreekumar, P., Sriram, S., Stalin, C.S., Sutaria, F., Sreedhar, Y.H., Barve, I.V., Mondal, C., Sahu, S.: In: Space Telescopes and Instrumentation 2016: Ultraviolet to Gamma Ray. Proc. SPIE, vol. 9905, p. 99051 (2016). 1608.01073. doi:10.1117/12.2235271

Wamsteker, W.: In: Morse, J.A., Shull, J.M., Kinney, A.L. (eds.) Ultraviolet-Optical Space Astronomy Beyond HST. Astronomical Society of the Pacific Conference Series, vol. 164, p. 261 (1999)

Wang, J., Meng, X.M., Han, X.H., Cai, H.B., Cao, L., Deng, J.S., Qiu, Y.L., Wang, S., Wei, J.Y., Hu, J.Y.: Astrophys. Space Sci. 360, 10 (2015). 1510.01435. doi:10.1007/s10509-015-2521-2

Wang, X.-Y., Huang, Z.-Q.: ArXiv e-prints (2017). 1712.03845

Werk, J.K., Prochaska, J.X., Tumlinson, J., Peeples, M.S., Tripp, T.M., Fox, A.J., Lehner, N., Thom, C., O'Meara, J.M., Ford, A.B., Bordoloi, R., Katz, N., Tejos, N., Oppenheimer, B.D., Davé, R., Weinberg, D.H.: Astrophys. J. 792, 8 (2014). 1403.0947. doi:10.1088/0004$637 \mathrm{X} / 792 / 1 / 8$ 